\title{
Program Design Principles to Support Teen-Adult Community Conservation Efforts
}

\begin{abstract}
Laura M. Cisneros ${ }^{1,2+*}$, Jonathan Simmons ${ }^{3 \dagger}$, Todd Campbell ${ }^{3 \dagger}$, Nicole Freidenfelds ${ }^{1}$, Chester Arnold ${ }^{4}$, Cary Chadwick ${ }^{4}$, David Dickson ${ }^{4}$, David Moss ${ }^{3}$, Laura Rodriguez ${ }^{5}$ and John C. Volin ${ }^{1,6,7}$
\end{abstract}

\begin{abstract}
${ }^{1}$ Department of Natural Resources and the Environment, University of Connecticut, Storrs, CT, United States, ${ }^{2}$ Institute of the Environment, University of Connecticut, Storrs, CT, United States, ${ }^{3}$ Department of Curriculum and Instruction, University of Connecticut Storrs, CT, United States, ${ }^{4}$ Department of Extension, University of Connecticut, Storrs, CT, United States, ${ }^{5}$ Department of Education, Eastern Connecticut State University, Willimantic, CT, United States, ${ }^{6}$ Office of the Provost, University of Maine, Orono, ME, United States, ${ }^{7}$ School of Forest Resources, University of Maine, Orono, ME, United States
\end{abstract}

Researchers and practitioners have identified numerous outcomes of place-based

OPEN ACCESS

Edited by:

Nancy Longnecker,

University of Otago, New Zealand

Reviewed by:

Cathy Cole,

University of Otago, New Zealand

Victoria Jane Metcalf,

Royal Society Te Apārangi,

New Zealand

*Correspondence:

Laura M. Cisneros

laura.cisneros@uconn.edu

${ }^{t}$ These authors have contributed equally to this work and share first authorship

Specialty section: This article was submitted to

STEM Education,

a section of the journal

Frontiers in Education

Received: 01 March 2021

Accepted: 17 August 2021

Published: 07 September 2021

Citation:

Cisneros LM, Simmons J, Campbell T, Freidenfelds N, Arnold C, Chadwick C,

Dickson D, Moss $D$, Rodriguez $L$ and Volin JC (2021) Program Design Principles to Support Teen-Adult Community Conservation Efforts.

Front. Educ. 6:674667. doi: 10.3389/feduc.2021.674667 environmental action (PBEA) programs at both individual and community levels (e.g., promoting positive youth development, fostering science identity, building social capital, and contributing to environmental quality improvement). In many cases, the primary audience of PBEA programs are youth, with less attention given to lifelong learners or intergenerational (e.g., youth and adult) partnerships. However, there is a need for PBEA programs for lifelong learners as local conservation decisions in the United States are often carried out by volunteer boards and commissions, which often have little formal conservation training. Intergenerational PBEA programs can provide an opportunity to bring together, in the case of this study, the unique skills and knowledge of teens (e.g., tech-savvy) and adults (e.g., knowledgeable of local community issues) that can lead to innovative ways of addressing real world endeavors that are relevant to participants and their communities.

This study describes a program model that offers structured learning opportunities that support intergenerational partnerships (teens and adults) as they contribute to community conservation efforts. We used a design-based research approach to develop and refine program design principles and communication pillars for the purpose of supporting successful teen-adult conservation projects, positive participant experiences, and science identity authoring. The principles and pillars drew on identity, cultural learning pathways, and community conservation research literature as well as previously collected participant interview data from our intergenerational PBEA program. We outline four design principles and four communication pillars that are critical to facilitate collaborative teenadult environmental action efforts and serve dual functions of providing program guidance and participant support. The aim of these principles and pillars are to establish collaborative team partnership norms that resist traditional hierarchical teen-adult relationships. Further, the principles and pillars consider how partners can draw on their interests, experiences, and knowledge of community, and utilize these assets along with conservation science disciplinary practices to accomplish meaningful 
science pursuits; thus facilitating how they identify themselves as contributing to science endeavors. Exemplar data and literature that support each principle and pillar are provided, and future extensions of these principles are discussed.

Keywords: communication pillars, community conservation, design principles, environmental action, science identity, intergenerational, lifelong learning, place-based

\section{INTRODUCTION}

The critical need for lifelong learners to participate in community conservation or place-based environmental action (PBEA) is motivated by the urgency to expand capacity to address emerging environmental issues (Horwich and Lyonm, 2007; Bonney et al., 2009; Ohmer et al., 2009; Short, 2010; Kransy, 2020), cultivate scienceliterate and civically engaged community members (Schusler et al., 2009; Short, 2010; Edwards, 2014; Kransy, 2020), and promote positive youth development and academic achievement (Schusler and Krasny, 2010; Schusler, 2015). Community conservation projects are efforts that are carried out by multiple community stakeholders that aim to protect, conserve, or improve local environments (Horwich and Lyon, 2007; Ohmer et al., 2009). PBEA programs support participants as they deliberately contribute to decision making, planning, implementation, and reflection of efforts intended to achieve a specific environmental outcome situated within their communities (Emmons, 1997; Schusler et al., 2009). Both participatory- and action-oriented approaches fall within the "democratic" paradigm of environmental education, aiming to enable learners to reflect upon and address social aspects of environmental problems that are relevant and meaningful to them (Schusler and Krasny, 2010). Examples of environmental action or community conservation include developing urban gardens in vacant lots to provide fresh produce to the community (Ohmer et al., 2009), erosion control along a stream bank in response to high levels of sedimentation (Tompkins, 2005), and monitoring black bearactivity patterns and habitat use in public areas to educate community members to reduce human-wildlife conflicts (e.g., Alegi et al., 2017).

Researchers and practitioners have identified numerous benefits of PBEA programs at both individual and community levels. These outcomes include promoting youth civic and professional development, fostering STEM (i.e., science, technology, engineering, and mathematics) identity and efficacy, developing a sense of place and nature connectedness, building social capital, and contributing to environmental quality improvement (Ohmer et al., 2009; Schusler et al., 2009; Short, 2010; Kransy, 2020; Rodriguez, 2020). Developing a sense of place can help participants feel a stronger connection to their environment, understand themselves as shapers of their environment (Ducre, 2013), and develop an appreciation for local resources; thus, combating deficit thinking about communities (Thomspon et al., 2020). Further, PBEA programs that specifically facilitate the co-design of the scientific or conservation project have a greater potential of meeting the needs of the community members, while also advancing conservation strategies (Golumbic et al., 2019; Senabre Hidalgo et al., 2021).

In many cases, the primary audience of PBEA programs are youth and adolescents, with less attention given to lifelong learners and intergenerational partnerships (Edwards, 2014; Peterson et al., 2019; Rodriguez, 2020). However, there is a need for PBEA programs for lifelong learners as conservation and land use decisions are often carried out at the local level by volunteer boards and commissions throughout the United States (Arnold, 2000; Nolon, 2014), which typically have little support in the form of education in natural resources or conservation science. PBEA programs that educate and partner adult conservation volunteers and adolescents may more effectively promote multiple outcomes of PBEA education. Specifically, intergenerational PBEA programs provide an opportunity to bring together the unique skills and knowledge of teens (e.g., tech-savvy) and adults (e.g., knowledgeable of local community environmental issues) that can lead to innovative ways of addressing real world pursuits and challenges that are relevant and important to the participants and their communities.

In this study, we describe design principles and communication pillars of a PBEA program model that offers structured learning opportunities to support intergenerational (teen and adult) community conservation efforts. Our work is situated within a design-based research (DBR) paradigm (Design-Based Research Collective, 2003), which provided a means to develop and refine program design principles and communication pillars that support the overarching goals of our PBEA program of promoting successful teen-adult volunteer environmental action efforts and intergenerational STEM identity authoring (e.g., the ways in which teens and adults come to view themselves as individuals who are capable of, willing to engage in, and have access to supportive social structures that recognize their skills and capabilities to contribute to meaningful STEM pursuits). Consequently, we outline four design principles and four communication pillars that have emerged from our work to date, describe the iterative designbased process undertaken for their development, and further detail and reify the principles and pillars with example case studies.

\section{METHODS}

\section{Learning Environment}

The University of Connecticut's Conservation Training Partnerships (UConn-CTP) (UConn NRCA CTP, 2021) is a NSF-funded STEM and PBEA program that uses an 

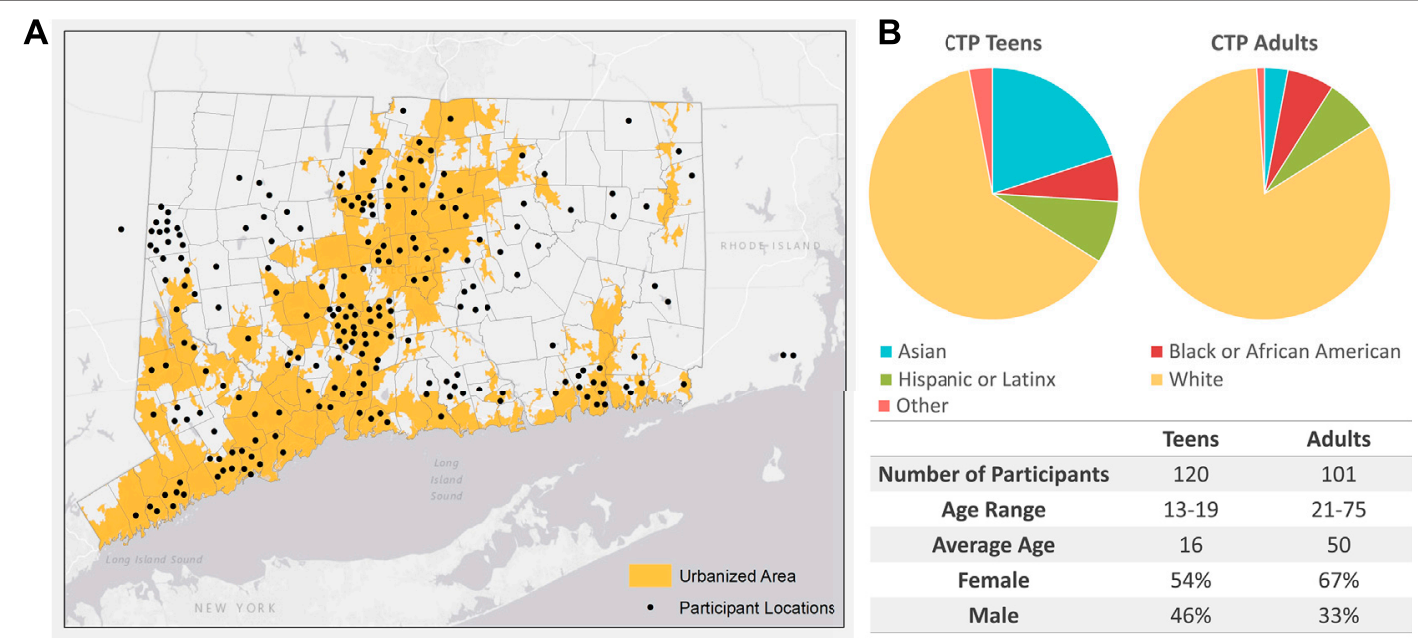

FIGURE 1 | (A) A distribution map showing the locations (black dots) of each UConn-CTP participant's community, which serves as the general location of each conservation project, throughout Connecticut and nearby states. Participants' communities fall along an urban (yellow shading) to rural (no shading) gradient, thus providing diverse context for community conservation projects (B) Demographic data from 221 UConn-CTP teen and adult participants.

A

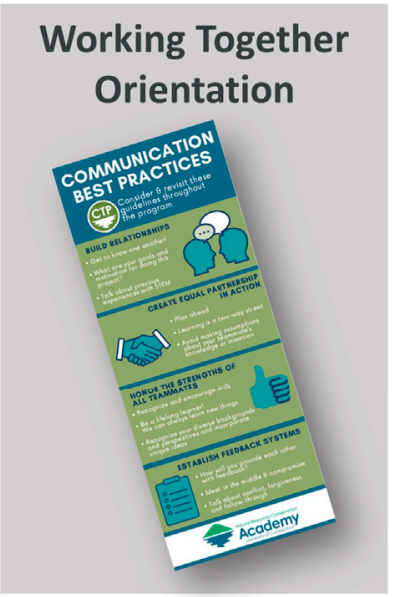

B

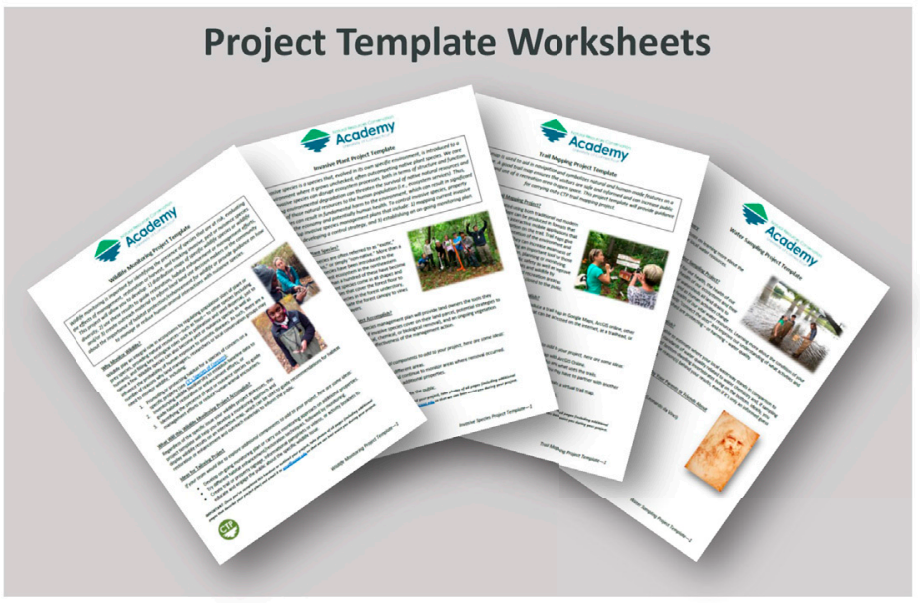

FIGURE 2 | Images of two types of participant project support documents (A) communication best practices to facilitate collaborative intergenerational teamwork, presented during a participant orientation and revisited throughout the program, and (B) project planning templates to provide a heuristic approach for scaffolding intergenerational team project development. See all original documents in the Supplementary Material.

intergenerational partnership framework to connect teens and adults from different backgrounds (Figure 1; e.g., participants from communities that span an urban to rural gradient) and empowers them to understand and address local environmental issues. First, intergenerational teams are formed prior to the start of the program (often recruited and formed by program faculty). These intergenerational teams comprise any combination of 1-4 high school students and 1-4 adults. Adults may be community or conservation volunteers (including land use decision makers from municipal commissions or land trusts), teachers, or family members, and may join the program with or without a prior connection to their teen teammates.
Next, the teen-adult teams attend a two-day immersive field workshop-held at different locations across Connecticut-where they begin to explore natural resource science concepts such as land use change, forest health, water resource protection, and biodiversity. Through hands-on field activities they explore how online mapping (geospatial) technology can be used to investigate conservation issues (Chadwick et al., 2018). Considerable time during the workshop is also dedicated to guiding the teen-adult teams through brainstorming and designing local conservation projects tailored to their interests and their community's needs. Through multiple iterations of the UConn-CTP 
workshops (10 total), we have developed web-based participant project support resources (Figure 2 and Supplementary Material; UConn CTP Resources, 2021). These include a past participant project showcase to orient new participants to the scope of conservation efforts undertaken by previous participant cohorts (UConn NRCA Projects, 2021), project planning templates (Figure 2B and Supplementary Material) that can be used as a heuristic approach for scaffolding initial conservation project development, and communications best practices to facilitate collaborative discussions and teamwork (Figure 2A and Supplementary Material). We note that the project planning templates are not meant to provide participants with a step-by-step approach to conducting a conservation project around a particular topic. Rather, the templates serve to provide guidance to participants about the different aspects of the project (e.g., locations of the project, timeline, disciplinary practices, and equipment/resources needed) they should consider in order to thoroughly developed their project tailored to their community needs and individual interests.

After the workshop, UConn-CTP teams carry out their community conservation projects throughout the summer, fall, and winter (most projects spanning from July to March). Projects are diverse and have included wildlife monitoring, trail mapping, invasive species management, water quality testing, and habitat restoration. Further, all projects employ one or more of the geospatial and conservation techniques learned during the workshop. UConn-CTP faculty provide significant postworkshop support through professional guidance, technical assistance, community connection, and access to a vast resource collection (UConn CTP Resources, 2021). Collectively, 221 teen and adult participants have carried out 71 community conservation projects throughout Connecticut since 2017 (Figure 1), with many UConn-CTP teams showcasing their work at a statewide environmental conference in March (see UConn NRCA Projects, 2021 to explore project topics and duration).

\section{Design-Based Approach}

The need for our design principles and communication pillars became apparent over time as we recognized the beneficial and challenging ways in which teens and adults engaged both with us and with each other during their community conservation projects (e.g., hierarchical relationships, different means of communicating). As such, the purpose of the principles and pillars was to support project completion, positive participant experiences, and STEM identity authoring.

Creation of the design principles and communication pillars was guided by design-based research (DBR) principles (DesignBased Research Collective, 2003), which embraces the connections between design and real-world contexts. This requires program designers to think flexibly about each aspect of the program, participant experiences and the relationship between program elements, design, and learning outcomes (Barab and Squire, 2004; Brown, 1992). We began by reflecting on the overarching goal of our STEM and PBEA program (UConn-CTP), which is to better understand intergenerational STEM identity authoring (e.g., the ways in which teens and adults come to view themselves as individuals who are capable of, willing to engage in, and have access to supportive social structures that recognize their skills and capabilities to contribute to meaningful STEM pursuits). Our DBR approach for the design principles and communication pillars is also guided by the high-level conjecture (Sandoval, 2004; Sandoval, 2014) or informed understanding that for participants to craft a STEM identity for themselves, intentionally designed structures and supports must exist for participants.

Initially, we used the following four considerations to propose design principles that could support intergenerational learners both in the workshop and in subsequent group work during their conservation projects:

1 The literature and our experience informed how we conceptualized intergenerational identity authoring happening in our program;

2 Previous program evaluations and informal observations stemming from over 70 UConn-CTP conservation projects;

3 Existing literature about informal STEM learning, STEM identity authoring, and cultural learning pathways; and

4 Prior experience with group interaction and project completion in classroom and university settings.

After articulating initial designs separately, the second and third authors came together to review and critique the collection of proposed design principles that emerged. When evaluating the initial proposed principles, the second and third authors continually reflected on STEM identity authoring and evaluated each proposed principle for its ability to help participants develop this view of themselves. It became clear that some principles overlapped while others did not. Additionally, some design principles were oriented to supporting intergenerational learners in developing their projects, while others focused on supporting productive communicative interactions among intergenerational teammates, all with the goal of developing STEM capabilities, recognition of these capabilities, and a supportive social network. This gave rise to the distinction between the design principles and communication pillars. The resultant design principles and communication pillars were subsequently shared with UConnCTP faculty and further refined for their ability to support program goals and enhance participant experience. Next, we used interview data from UConn-CTP intergenerational teams ( 8 teens and 7 adults) that were collected at multiple points during and after the completion of the intergenerational team's conservation project to study intergenerational identity authoring (Rodriguez et al., 2020; Rodriguez, 2020; Campbell et al., 2021; Simmons et al., in review). These data were used to determine the extent to which each design principle could be mapped to previous benefits or challenges referenced by participants.

After evidence of the need for, and benefit of, each principle was established by the literature and participant data, the design principles and communication pillars were subsequently mapped 


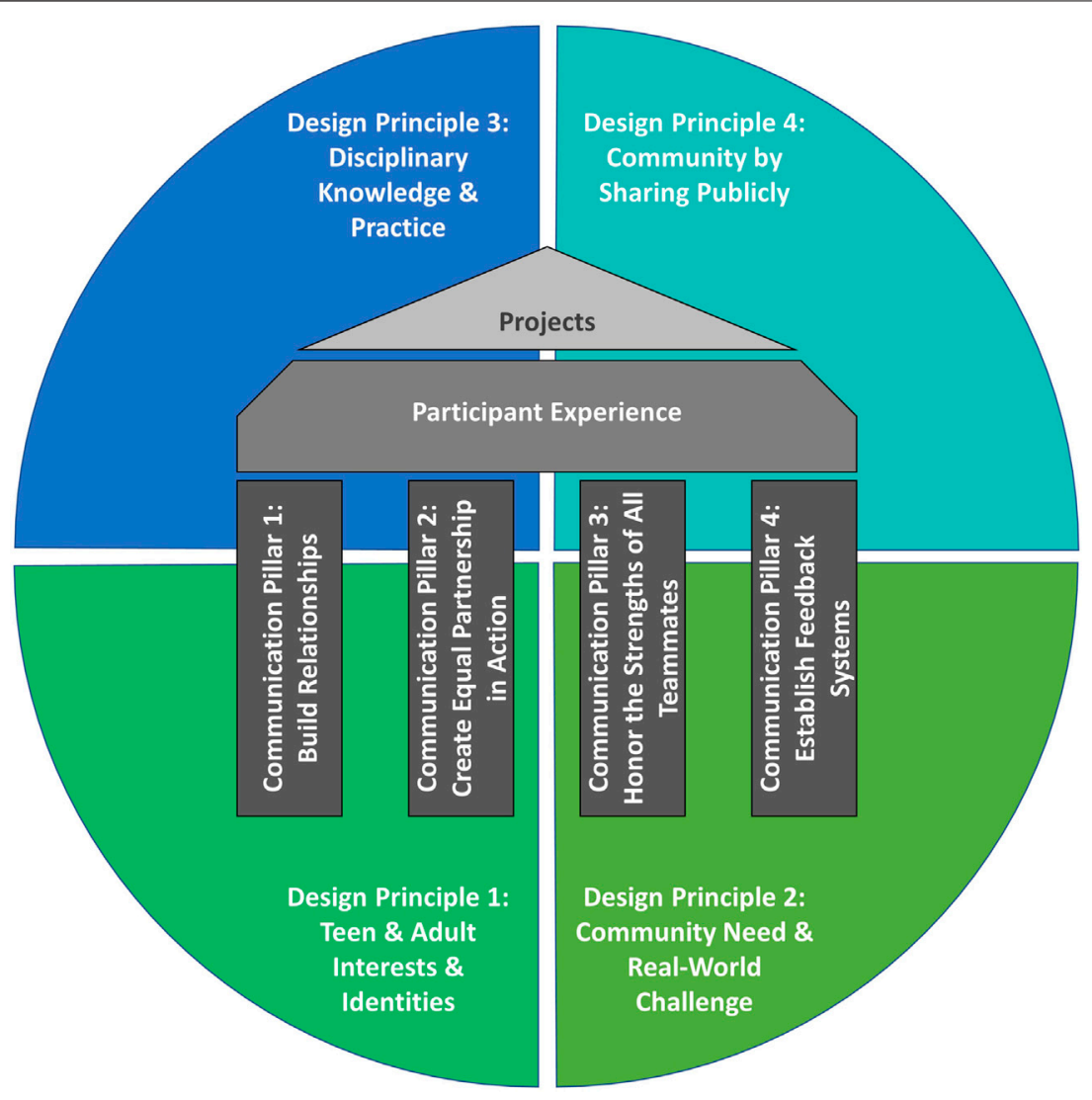

FIGURE 3 | Diagram of our program design principles and communication pillars that facilitate collaborative intergenerational community conservation projects. The principles and pillars serve dual functions of guiding program features and providing participant support. As such, the four design principles (underlying circle) guide all our program strategies. The four communication pillars support the positive experiences of our participants by disrupting traditional uneven power structures in intergenerational partnerships and providing strategies for collaborative team norms, thus allowing all teammates to engage in conservation work more deeply together.

to program features, such as the project planning templates (Figure 2 and Supplementary Material) and workshop or conservation project facilitation strategies to determine where these principles and pillars already exist or where they might be added to further improve participants' STEM identity authoring and successful community conservation efforts.

\section{RESULTS}

\section{Design Principles}

The four design principles encourage our participants to connect their projects to: 1) both partners' interests and identities, 2) community needs and real-world challenges, 3) current disciplinary knowledge and practices, and 4) community by sharing it publicly at local events or conferences (Figure 3). Below, we describe each design principle and support them with 1) relevant literature, 2) case studies that exemplify how the design principles are taken up in participant projects, and 3) participant interview data that demonstrate the importance of each principle. We note that the quotations used the Participant Interview Data sections do not always come from the participants described in the Case Study sections in order to provide additional support for each principle.

\section{Design Principle 1: Connect Project to Teen and Adult Interests and Identities}

The first principle encourages both teen and adult participants to connect their community conservation project to their prior experiences. This helps ensure that the project draws on the assets of both partners, avoiding deficit framing and allowing for more expansive and meaningful ways for participants to engage in science and conservation. This asset-based approach facilitates STEM identity authoring (Rodriguez et al., 2020, Rodriguez, 2020), which in turn can promote lifelong STEM learning and participation (Carlone \& Johnson, 2007).

\section{Supporting Literature}

This design principle finds its roots in the identity and cultural learning pathways literature. Here, identity can be understood as the negotiated self-narrations or self-construals individuals and others use to answer questions about who an individual is (Lee, 2017). A STEM identity refers to how a person identifies with a STEM field and is recognized as being a person who belongs in 
and is capable of understanding and applying disciplinary concepts, participating in pursuits of consequence, and contributing to that field. The development of a STEM identity is important in deepening an interest in a STEM field into perseverance in the field (Carlone and Johnson, 2007). Additionally, as Meyer (1998) notes, "knowing something, then, is a cultural experience that strengthens or fractures culture," thus culture must be considered and shared as STEM identity is developed (p. 22). Here, culture can be understood more broadly in alignment with Bang et al. (2017) as, "ways of knowing, talking, valuing, and acting as we live out our day-today lives inside family and community" recognizing that "human beings make sense of the world in ways that are both similar and different" (p. 35). Consequently, cultural learning pathways theory (Bell et al., 2012) considers the multidimensionality, fluidity, and plurality of identity authorship (Barton and Tan, 2010; Vareles et al., 2012). Here, a cultural learning pathway recognizes that learning happens across space and time over a lifespan in the pursuit of personal goals. Central to cultural learning pathways are how interests can launch, be strengthened, and shape identity through situated events that help individuals (i.e., teens and adults) negotiate their selfconstruals of who they are. Given this, as a way to support identity authoring, this design principle prioritizes connecting the focus of participants' community conservation efforts to the interests and identities of teens and adults.

\section{Case Study 1: Geologic Natural History of a State Park}

The following case study demonstrates how intergenerational teams can incorporate science and environmental interests as well as other non-science interests such as art, technology, and other extracurricular interests, into their conservation projects. During a project brainstorming exercise, the intergenerational team in this example who did not know each other prior to the program-discussed the teen's interest in geology and the adult's enjoyment of hiking and connection to state parks as an environmental state agency employee. They combined their unique interests to create an online interactive map of a popular state park trail so that the public can take a virtual hiking tour and learn about various geological features along the trail. This team also leveraged their interest in photography to incorporate a creative element to their project by including photographs in pop-up windows that interpreted what each geological feature resembled. For example, one rock outcrop was compared to an elephant and another to a volcano. This is just one illustration of how we can allow participants to explore expansive ways of engaging in environmental efforts and afford participants opportunities to draw on their multiple intersecting identities in completing their projects.

\section{Participant Interview Data Supportive of Design Principle 1}

- "Do something you care about. . . just do something fun, and do something that you enjoy, and that you're passionate about." UConn-CTP Teen

- "I was interested in learning more about the technology aspect and working on another project that involved environmental issues because I'm really interested in that kind of stuff. . . so I can map out different areas of trails and things like that, where I find interesting things to come back to." UConn-CTP Teen

\section{Design Principle 2: Connect Project to Community Need and Real-World Challenges}

Through principle 2, participants connect their project to a community need and a real-world challenge. Not only does this set up the project to have real community benefits, but it provides participants with the opportunity to see the power in applying their disciplinary and action-oriented knowledge in the context of addressing community issues that are relevant to them, further consolidating the first principle.

\section{Supporting Literature}

Recently, STEM education and citizen science researchers have noted the importance of engaging learners in meaningful pursuits, like explaining real-world phenomena or solving problems of consequence (National Research Council, 2013; Krajcik, 2015; Golumbic et al., 2019; San Llorente Capdevila et al., 2020; Senabre Hidalgo et al., 2021). Other social scientists (e.g., Vygotsky, 1987) have noted the importance of a focus on why people are engaged in activities or the meaningfulness of pursuits (e.g., to solve a conservation problem; explain a realworld phenomenon), since these pursuits provide a framework for what competences or performances matter and why (Hyysalo, 2005). In this design principle, we prioritize connecting teen and adult projects to community need and real-world challenges to both draw on a combination of notions of relevancy and authenticity in supporting learners in informal learning contexts (Dierking et al., 2003) and to support teens and adults in deliberately contributing to decision making, planning, implementation, and reflection to achieve a specific environmental outcome situated within their communities (Emmons, 1997; Schusler et al., 2009; Golumbic et al., 2019; Senabre Hidalgo et al., 2021). This is important, since Rivera Maulucci et al. (2014) argue, that when we ground learning and participation "in students" (and adults') lives, their identities develop in the context of exploring problems that are meaningful to them and to their communities" (p. 1123). Further, when connecting projects to community, it is critical that community voice is recognized and honored, and that any project aligns with community values (Metcalf and Style, 2019).

\section{Case Study 2: Urban Tree Reuse Project}

In this case study, the teen-adult team did not know each other before joining UConn-CTP but lived in the same urban community. The student had an interest in forestry and the adult partner, as a manager of a park sustainability program, was aware of city trees scheduled for removal after having been infected by emerald ash borer beetles. Through further research, the team recognized that the proposed wood chipping method for wood disposal would result in the loss of an important source of carbon storage and a valuable natural resource product. Together they developed an urban tree reuse project where they worked with the city to recuperate the ash wood, crafting beautiful benches that were then placed back into 
local parks. This participant project has continued to be active beyond the support of the UConn-CTP program.

Participant Interview Data Supportive of Design Principle 2

- "We've been going along with our removing the dead trees, reusing the lumber, and then replacing those trees. It's a whole lot of conservation and community benefits." UConn-CTP Teen

- "Going through this project and being with it for such a long time and seeing it come to fruition, and having all these grants and stuff, like coming into play, it's like it gives me a deeper connection to my local community." UConnCTP Teen

- "Seeing this create this opportunity for kids to get involved in their own community, and make those connections with leaders in the community and the local government or anything like that, is just awesome." UConn-CTP Adult

\section{Design Principle 3: Connect Project to Disciplinary Knowledge and Practice}

Principle three encourages participants to utilize knowledge and practices of experts when planning and implementing their conservation projects. These connections allow for partnerships with a range of people from a variety of fields, including scientists and community organization leaders.

\section{Supporting Literature}

This design principle is shaped by social practice that happens in what Lave and Wenger (1991) and Wenger (1998) referred to as "communities of practice" where identity is authored (Carlone and Johnson 2007). Communities of practice can be understood as groups of individuals with common interests (e.g., hobbyists) or engaged in common forms of activity (e.g., naturalists) who over time have developed competencies (i.e., knowledge) and practices (i.e., ways of working at knowing or solving problems) supportive of accomplishing their pursuits. Gee (2000-2001) points to how engaging in a community of practice, initially on the periphery and more centrally over time, shapes the "kind of person" one is seeking to be and enact. In this, there is a recognition that one cannot successfully enact a particular identity that is legitimized by oneself and others without drawing on relevant competencies and practices that are suited for meeting group-level or a community of practice's needs. As such, this design principle aims to connect teen and adult learners to communities of practice (e.g., amateur birders) to both leverage disciplinary knowledge and practice to accomplish their desired pursuits, while also connecting them to communities where identities are constructed. Supporting structures in the way of communities of practice and training on disciplinary practices and protocols is also a key factor to for successful citizen science projects (Liberatore et al., 2018; San Llorente Capdevila et al., 2020).

\section{Case Study 3: The Beavers of Mendell's Folly}

This teen-adult duo wanted to highlight the importance of a beaver-created wetland on a land trust property. The adult partner was a land trust volunteer, and her teen partner's former middle school teacher. For the project, they researched scientific literature and reached out to several relevant experts to gain insight and understanding about the role of beavers as ecosystem engineers. For example, they toured the University of Connecticut's Biodiversity Research Collections to learn more about local wetland-associated mammals, and conducted interviews with a graduate student studying wetlands and a biologist at a nearby nature center. They integrated these varied resources into an Esri StoryMap (geospatial technology taught at the UConn-CTP workshop), which allowed them to convey a multitude of information using a storytelling strategy-including text, multimedia, and maps-to engage and inspire a broader audience. The value of connecting their project to disciplinary knowledge and practice was evinced by an award from the 2020 EsriUser Conference Student Map Competition for their StoryMap ( $\mathrm{Lu}$ and Arnini, 2020).

Participant Interview Data Supportive of Design Principle 3

- "Why should we just chip up the wood and put it in the landfill where all that carbon eventually goes back into the atmosphere when we can use that wood to create furniture, like a bench or a chair, that will keep that carbon sequestered longer and have this extra benefit of just being an awesome piece of furniture." UConn-CTP Teen

- "I have definitely gotten a bit better at birds. I can like transfer grips, and I can hold birds better. . . I learned about stopover sites, which was something like-it can be extrapolated from knowing anything about migration, but I hadn't really learned about, in detail." UConn-CTP Teen

\section{Design Principle 4: Connect Project to Community by Sharing Publicly}

The fourth principle of sharing the project with the community and broader public serves three goals: 1) it informs the public about community members who are actively engaged in community improvements, 2) it publicly recognizes the accomplishment of both partners, and 3) it highlights the opportunity for other community members to contribute. Similar to design principles 2 and 3, this principle has a dual purpose of both supporting teen and adult identity authoring and supporting community conservation efforts. While UConn-CTP project final products may range from a poster, article in a local newspaper, an Esri StoryMap, or a park bench, teen and adult participants are encouraged and supported to share their projects publicly. For many, this means presenting at a statewide conservation conference as well as sharing locally via in-person events (e.g., town halls, public fairs, community outreach events at land trusts, libraries or schools) or through online/social media platforms of local community organizations.

\section{Supporting Literature}

Public communication of local environmental efforts by community members is essential to bring awareness of issues where they matter most as well as build social capital among community members (Conrad and Daoust, 2008). Increased 
social capital within a community can play a key role in increasing and sustaining stakeholder involvement in future community efforts (Conrad and Daoust, 2008). Most important in relation to identity authoring, this design principle aims to afford teens and adults recognition. Recognition is important since it can be understood in relation to identity as the juxtaposition of a person's internal designations (how they see themself) and the social designations ascribed by others (how they are seen by others) (Carlone and Johnson, 2007; Hazari et al., 2015).

\section{Case Study 4: Municipal Water Conservation Education}

One daughter-mother team focused their conservation efforts on aiding their town's application to become a certified "SustainableCT" town. They contributed to this effort by developing and distributing a survey to better understand community members' views on water conservation, which later guided water conservation educational materials distributed to town residents. As such, this team authored several articles in local news outlets, both online and in print, to reach town residents more broadly to distribute the survey. They also presented the results of the project at a town council meeting so that their findings could be integrated into the town's sustainability initiatives. Following the UConn-CTP program, the teen continued her efforts, and paired with an elementary school to provide water conservation education to children. Through this example we aim to emphasize the importance of external recognition in building participants' confidence and identity such that they feel capable of continuing their conservation work beyond our program.

Participant Interview Data Supportive of Design Principle 4

- "During that project, I published a few articles in the local newspapers about why we should care about this issue. That was really enjoyable to me spreading awareness, so I continued to try and write articles about conservation in general and send them to local newsletters." UConnCTP Teen

- "I think people were really interested in what I had to say. I was a bit surprised by how open and supportive and interested people were, especially 'cause I was a young person presenting to these all the people that came to the event were basically older adults and I was just I didn't think they were gonna take me as seriously as I thought they as they actually did." UConn-CTP Teen

- "Oh, it was a very proud moment to see her with three or four other presenters from different fields, from the water company, the town selectman,... the sustainable board, the town's sustainable advisory board, and a couple of other participants who also presented." UConn-CTP Adult

\section{Communication Pillars}

The communication pillars were developed to support productive interactions among intergenerational teammates (Figure 3). These pillars were introduced to intergenerational teams at the beginning of the summer workshop. Participants were asked to consider them often and revisit them throughout the program in relation to their interactions with their teen or adult partner. Each pillar is introduced briefly, alongside example participant quotes indicative of how each can manifest in the interactions among intergenerational teammates.

\section{Communication Pillar 1: Build Relationships}

Participants are shaped by their culture and previous experiences with STEM. Taking the time to explore participants' interests and experiences strengthened their connection to the project and helped strengthen their STEM identities. Further, the relationship building process allowed participants to build trust in each other, which was critical to the success of their project. The following prompts introduced participants to this pillar:

- Get to know one another!

- What are your goals and motivation for doing this project?

- Talk about previous experiences with STEM.

\section{Participant Interview Data Indicative of Communication Pillar 1}

- "It's nice to see her grow as a student. She didn't say a word to me the first time we met, by the way. She's very quiet. Seeing her grow as a person who felt comfortable telling me something as simple as, "I think the wording should be different here," she wouldn't have told me that back in the summer." UConn-CTP Adult

- "I want to learn what she knows. She says she's a bird expert or something. I just want to learn more science. I'm not-this is all really new to me." UConn-CTP Teen

- "Now knowing more about her personal interests, more about what she's interested in doing with her life, I can better support that." UConn-CTP Adult

\section{Communication Pillar 2: Create Equal Partnership in Action}

The intergenerational partnership aspect of UConn-CTP is designed to disrupt traditional teen-adult interactions where power disproportionately resides with adults. Reframing this experience as a learning experience for both partners and planning for an equal partnership shaped the way intergenerational teams interacted and how power was more evenly distributed across teens and adults. The following prompts introduced participants to this pillar:

- Plan ahead.

- Learning is a two-way street.

- Avoid making assumptions about your teammate's knowledge or intentions.

\section{Participant Interview Data Indicative of Communication Pillar 2 \\ - "From that point of view, we were well-matched because nobody's feeling like they are overwhelmed or aren't doing enough." UConn-CTP Adult}


- "A thing I do like in science that it is a team effort, not like, it's not like math, or where you have to work independently. I like the different ideas coming across, so what (one partner) knows, I might not know, what (another partner) knows, I still might not know, or what they don't know, I can enlighten them, so I feel I love the team effort, and its collaboration." UConn-CTP Teen

\section{Communication Pillar 3: Honor the Strengths of All Teammates}

Participants bring a diverse set of prior experiences and a range of knowledge and ways of knowing to their project. Recognizing the knowledge, ways of knowing, and strength of an individual participant bolstered their STEM identity and allowed them to incorporate unique ideas to find innovative solutions. The following prompts introduced participants to this pillar:

- Recognize and encourage skills.

- Be a lifelong learner. We can always learn new things.

- Recognize your diverse backgrounds and perspectives and incorporate unique ideas.

\section{Participant Interview Data Indicative of Communication Pillar 3}

- "I think we both share listening to each other's ideas. . I I don't feel like either one of us tries to take the lead. I feel like depending on what the task is. Because it's the bench and Marcus (pseudonym) has made one before, he may take the lead in that portion, but if it's, say, it's using a tool, or sanding, or measuring, or cutting, or something that I've done before or am comfortable with, he allows me to take the lead on that." UConn-CTP Adult

- "I'm trying to put what I already know into the project. I'm trying the best that I can. She knows a lot more than I do. I'm trying to understand, and I don't want to make her do all the work. I want to be able to help." UConn-CTP Teen

\section{Communication Pillar 4: Establish Feedback Systems}

Effective teamwork relies on effective communication. This is especially important since individuals of different ages and cultures have different expectations about communication, collaboration, and perceptions of time that can potentially lead to conflict if not planned for when making explicit guidance for communication and feedback. This is important, since participants may make assumptions about the value or intention of their teammates that negatively impacts their impression of their teammate. Through establishing feedback systems early in the process, participants had a principled plan for communication. The following prompts introduced participants to this pillar:

- How will you provide each other with feedback?

- Meet in the middle and compromise.

- Talk about conflict, forgiveness, and follow-through.

\section{Participant Interview Data Indicative of Communication}

\section{Pillar 4}

- "I think that was a big having to learn how to communicate effectively within our team and how to reach out. . It was a good experiment in leadership and management and also just understanding what was best for the overall project and the team." UConn-CTP Adult

- "I will say that the students definitely kept me on track, especially Jackie [pseudonym]. "Cause she would text me, like, "I haven't heard from you about this." UConn-CTP Adult

- "I feel like the only problem there was communication just “cause we didn't really use any of the same mediums. Max and I would text or Snapchat each other and then we'd email Jillian (pseudonyms). It was kind of hard to organize everything." UConn-CTP Teen

\section{CONCLUSION AND FUTURE DIRECTIONS}

In our place-based environmental action program work to date, we have tried to design a program that addresses the critical need for lifelong learners, both teens and adults, to engage meaningfully in environmental action to address the urgent need for expansive local approaches to environmental issues (Horwich and Lyon, 2007; Ohmer et al., 2009; Short, 2010; Kransy, 2020) and support the fluid and intersectional STEM identity authorship of intergenerational learners (Rodriguez et al., 2020; Rodriguez, 2020). As reported, we have done this by supporting 221 teen and adult participants to carry out over 71 community conservation projects throughout Connecticut and nearby states since 2017 (Figure 1). Along the path to supporting the successful completion of these projects, we engaged in an adapted iterative DBR approach (Design-Based Research Collective, 2003) to develop and refine the program design principles and communication pillars that drew on existing research from identity theory (e.g., Gee, 2000-2001), cultural learning pathways (e.g., Bell et al., 2012), activity theory (e.g., Vygotsky, 1987), and community conservation research (e.g., Horwich and Lyon 2007; Ohmer et al., 2009). This literature foundation was considered alongside our previous experience as STEM and environmental educators and examined in the context of previously collected interview data. In the end, the formation of these design principles and communication pillars highlighted the need for creating space for participants to share their backgrounds and experiences and for program materials that made space for their culture in the planning process of their conservation projects.

While we have been able to establish the validity and usefulness of the design principles and communication pillars, we share these as the most recent iterations that we will continue to refine and improve, as design-based research is a cyclical process that requires testing and iteratively modifying interventions within real-world contexts to develop practical approaches. These principles and pillars are important foundations for undertaking aims of equitable participation 
within intergenerational community conservation efforts. They rely on STEM identity and cultural learning pathways research. We recognize that these design principles do not yet fully consider the environmental justice and anti-racist aims which we aspire to continue to learn about and center in our PBEA programs. Given this, we see our work as ongoing and invite others to engage critically with us as we seek to meet the goals of centering equity, inclusion, and belonging within PBEA programs.

\section{DATA AVAILABILITY STATEMENT}

The raw data supporting the conclusions of this article will be made available by the authors, without undue reservation.

\section{ETHICS STATEMENT}

The studies involving human participants were reviewed and approved by Institutional Review Board, University of Connecticut. Written informed consent to participate in this study was provided by the participants' legal guardian/next of kin.

\section{AUTHOR CONTRIBUTIONS}

LC, NF, CA, CC, DD and JV developed and implemented multiple iterations of the UConn-CTP program, with guidance by TC, DM, LR and JS. JS and TC developed the conceptual framework of the design principles and communication pillars, and used previously collected interview data collected by LR and

\section{REFERENCES}

Alegi, E., Somogie, G., and Kane, L. (2017). The Effects of Increasing bear Activity at the Bent of the River. Available at: https://nrca.uconn.edu/projects/posters/ cap_2016_73.pdf (Accessed February 28, 2021).

Arnold, C. L. (2000). Land Use Is the Issue, but Is Land Grant the Answer? J. Ext. 38, 6COM1.

Bang, M., Brown, B. A., Calabrese Barton, A., Rosebery, A. S., and Warren, B. (2017). in "Toward More Equitable Learning in Science" in Helping Students Make Sense of the World Using Next Generation Science and Engineering Practices. Editors C. Schwarz, C. Passmore, and B. J. Reiser (Arlington, VA: National Science Teachers Association)), 33-58.

Barab, S., and Squire, K. (2004). Design-based Research: Putting a Stake in the Ground. J. Learn. Sci. 13, 1-14. doi:10.1207/s15327809jls1301_1

Barton, A. C., and Tan, E. (2010). We Be Burnin'!Agency, Identity, and Science Learning. J. Learn. Sci. 19, 187-229. doi:10.1080/ 10508400903530044

Bell, P., Tzou, C., Bricker, L., and Baines, A. D. (2012). Learning in Diversities of Structures of Social Practice: Accounting for How, Why and where People Learn Science. Hum. Dev. 55, 269-284. doi:10.1159/000345315

Bonney, R., Cooper, C. B., Dickinson, J., Kelling, S., Phillips, T., Rosenberg, K. V., et al. (2009). Citizen Science: A Developing Tool for Expanding Science Knowledge and Scientific Literacy. Bio Sci. 59, 977-984. doi:10.1525/ bio.2009.59.11.9

Brown, A. L. (1992). Design Experiments: Theoretical and Methodological Challenges in Creating Complex Interventions in Classroom Settings. J. Learn. Sci. 2, 141-178. doi:10.1207/s15327809jls0202_2
JS, with assistance by LC and NF. All authors contributed to the refinement of the design principles and communication pillars. JS, TC, LC and NF mapped and added the design principles and communication pillars into existing participant project support documents. LC, JS, TC, and NF led the writing of this paper, with additional contributions from CA, CC, DD, DM, LR and JV.

\section{FUNDING}

Funding for the UConn-CTP program is provided by the National Science Foundation Advancing Informal STEM Learning program under Grant No. 1612650. Any opinions, findings, and conclusions or recommendations expressed in this material are those of the authors and do not necessarily reflect the views of the National Science Foundation.

\section{ACKNOWLEDGMENTS}

We wish to thank external evaluators Dr. Joan Pasley and Dr. Keith Esch from Horizon Research, Inc. for their guidance and feedback on the UConn-CTP education programming and integrated research.

\section{SUPPLEMENTARY MATERIAL}

The Supplementary Material for this article can be found online at: https:/www.frontiersin.org/articles/10.3389/feduc.2021.674667/ full\#supplementary-material

Campbell, T., Rodriguez, L., Moss, D. M., Volin, J. C., Arnold, C., Cisneros, L., et al. (2021). Intergenerational Community Conservation Projects, STEM Identity Authoring, and Positioning: the Cases of Two Intergenerational Teams. Int. J. Sci. Educ. B 11, 174-190. doi:10.1080/21548455.2021.1923081

Carlone, H. B., and Johnson, A. (2007). Understanding the Science Experiences of Successful Women of Color: Science Identity as an Analytic Lens. J. Res. Sci. Teach. 44, 1187-1218. doi:10.1002/tea.20237

Chadwick, C., Dickson, D., Arnold, A., Cisneros, L., Volin, J., Campbell, T., et al. (2018). Connecting Generations through Informal Geospatial and Conservation Education. J. Ext. 56, 5IAW2.

Conrad, C. T., and Daoust, T. (2008). Community-based Monitoring Frameworks: Increasing the Effectiveness of Environmental Stewardship. Environ. Manage. 41, 358-366. doi:10.1007/s00267-007-9042-x

Design-Based Research Collective (2003). Design-based Research: An Emerging Paradigm for Educational Inquiry. Educ. Res. 32, 5-8. doi:10.3102/ 0013189x032001005

Dierking, L. D., Falk, J. H., Rennie, L. o., Anderson, D., and Ellenbogen, K. (2003). Policy Statement of the ?informal Science Education? Ad Hoc Committee. J. Res. Sci. Teach. 40, 108-111. doi:10.1002/tea.10066

Ducre, K. A. (2013). A Place We Call Home: Gender, Race, and Justice in Syracuse. New York: Syracuse University Press.

Edwards, R. (2014). Citizen Science and Lifelong Learning. Stud. Educ. Adults 46, 132-144. doi:10.1080/02660830.2014.11661662

Emmons, K. M. (1997). Perspectives on Environmental Action: Reflection and Revision through Practical Experience. J. Environ. Educ. 29, 34-44. doi:10.1080/ 00958969709599105

Gee, J. P. (2000-2001). Identity as an Analytic Lens for Research in Education. Rev. Res. Educ. 25, 99-125. doi:10.2307/1167322 
Golumbic, Y. N., Fishbain, B., and Baram-Tsabari, A. (2019). User Centered Design of a Citizen Science Air-Quality Monitoring Project. Int. J. Sci. Educ. Part B 9, 195-213. doi:10.1080/21548455.2019.1597314

Hazari, Z., Cass, C., and Beattie, C. (2015). Obscuring Power Structures in the Physics Classroom: Linking Teacher Positioning, Student Engagement, and Physics Identity Development. J. Res. Sci. Teach. 52, 735-762. doi:10.1002/ tea. 21214

Horwich, R. H., and Lyon, J. (2007). Community Conservation: Practitioners' Answer to Critics. Oryx 41, 376-385. doi:10.1017/s0030605307001010

Hyysalo, S. (2005). Objects and Motives in a Product Design Process. Mind, Cult. Activity 12, 19-36. doi:10.1207/s15327884mca1201_3

Krajcik, J. (2015). Three-dimensional Instruction: Using a New Type of Teaching in the Science Classroom. Sci. Child. 53, 6-8. doi:10.2505/4/sc15_053_03_6

Kransy, M. E. (2020). Advancing Environmental Education Practice. Ithaca, New York: Cornell University Press.

Lave, J., and Wenger, E. (1991). Situated Learning: Legitimate Peripheral Participation. Cambridge, UK: Cambridge University Press.

Lee, C. D. (2017). Expanding Visions of How People Learn: The Centrality of Identity Repertoires. J. Learn. Sci. 26, 517-524. doi:10.1080/ 10508406.2017.1336022

Liberatore, A., Bowkett, E., MacLeod, C., Spurr, E., and Longnecker, N. (2018). Social media as a Platform a Citizen Science Community of Practice. Citizen Sci. Theor. Pract. 3, 1-14. doi:10.5334/cstp.108

Lu, M., and Arnini, C. (2020). The Beavers of Mendell's Folly. Available at: https:// storymaps.arcgis.com/stories/a0942949cf204a088f6d0421056e1026 (Accessed February 22, 2021).

Metcalf, V. J., and Style, R. L. (2019). Cultural Considerations in Citizen Health Science and the Case for Community-Based Approaches. Am. J. Bioeth. 19, 40-43. doi:10.1080/15265161.2019.1619874

Meyer, M. A. (1998). Native Hawaiian Epistemology: Sites of Empowerment and Resistance. Equity Excell. Educ. 31, 22-28. doi:10.1080/1066568980310104

National Research Council (2013). A Framework for K-12 Science Education: Practices, Crosscutting Concepts, and Core Ideas. Washington, DC: National Academies Press.

Nolon, J. R. (2014). Protecting the Environment through Land Use Law: Standing Ground. Washington, DC: Environmental Law Institute.

Ohmer, M. L., Meadowcroft, P., Freed, K., and Lewis, E. (2009). Community Gardening and Community Development: Individual, Social and Community Benefits of a Community Conservation Program. J. Community Pract. 17, 377-399. doi:10.1080/10705420903299961

Peterson, M. N., Stevenson, K. T., and Lawson, D. F. (2019). Reviewing How Intergenerational Learning Can Help Conservation Biology Face its Greatest challenge. Biol. Conservation 235, 290-294. doi:10.1016/j.biocon.2019.05.013

Rivera Maulucci, M. S., Brown, B. A., Grey, S. T., and Sullivan, S. (2014). Urban Middle School Students' Reflections on Authentic Science Inquiry. J. Res. Sci. Teach. 51, 1119-1149. doi:10.1002/tea.21167

Rodriguez, L. S., Morzillo, A., Volin, J. C., and Campbell, T. (2020). Conservation Science and Technology Identity Instrument: Empirically Measuring STEM Identities in Informal Science Learning Programs. Sch. Sci. Maths. 120, 244-257. doi:10.1111/ssm.12401

Rodriguez, R. (2020). STEM Identity Authoring: Intergenerational Collaborative Learning in Informal STEM Programs [dissertation]. [Storrs (CT)]: University of Connecticut.

San Llorente Capdevila, A., Kokimova, A., Sinha Ray, S., Avellán, T., Kim, J., and Kirschke, S. (2020). Success Factors for Citizen Science Projects in Water Quality Monitoring. Sci. Total Environ. 728, 137843. doi:10.1016/ j.scitotenv.2020.137843

Sandoval, W. A. (2004). Developing Learning Theory by Refining Conjectures Embodied in Educational Designs. Educ. Psychol. 39, 213-223. doi:10.1207/ s15326985ep3904_3

Sandoval, W. (2014). Conjecture Mapping: An Approach to Systematic Educational Design Research. J. Learn. Sci. 23, 18-36. doi:10.1080/ 10508406.2013.778204
Schusler, T. M. (2015). in "Environmental Action and Positive Youth Development," in across the Spectrum. Editors M. C. Monroe and M. E. Krasny (Washington, DC: North American Association for Environmental Education)), 142-163.

Schusler, T. M., and Krasny, M. E. (2010). Environmental Action as Context for Youth Development. J. Environ. Educ. 41, 208-223. doi:10.1080/ 00958960903479803

Schusler, T. M., Krasny, M. E., Peters, S. J., and Decker, D. J. (2009). Developing Citizens and Communities through Youth Environmental Action. Environ. Educ. Res. 15, 111-127. doi:10.1080/13504620802710581

Senabre Hidalgo, E., Perelló, J., Becker, F., Bonhoure, I., Legris, M., and Cigarini, A. (2021). "Participation and Co-creation in Citizen Science," in The Science of Citizen Science. Editors K. Vohland, A. Land-Zandstra, L. Ceccaroni, R. Lemmens, J. Perelló, M. Ponti, et al. (Berlin, Germany: Springer), 199-218. doi:10.1007/978-3-030-58278-4_11

Short, P. C. (2010). Responsible Environmental Action: its Role and Status in Environmental Education and Environmental Quality. J. Environ. Educ. $41,7-21$.

Simmons, J., Campbell, T., Moss, D. M., Volin, J., Arnold, C., Cisneros, L. M., et al. Preparation.Part of Our DNA": Intergenerational Family Learning in Informal Science International Journal of Science Education. In Review.

Thompson, J., Mawyer, K., Johnson, H., Scipio, D., and Luehmann, A. (2020). "Culturally and Linguistically Sustaining Approaches to Ambitious Science Teaching," in Preparing Science Teachers through Practice-Based Teacher Education. Editors D. Stroupe, K. Hammerness, and S. McDonald (Boston, MA: Harvard Press), 45-61.

Tompkins, L. (2005). A Case for Community-Based Education: Students Form Partnerships to Tackle Local Environmental Issues. Sci. Teach. 72, 34-36.

UConn CTP Resources (2021). University of Connecticut's Conservation Training Partnerships Program Materials. Available at: https://nrca.uconn. edu/students-adults/materials.htm (Accessed February 28, 2021).

UConn NRCA CTP (2021). University of Connecticut's Conservation Training Partnerships Program. Available at: https://nrca.uconn.edu/students-adults/ index.htm (Accessed February 28, 2021).

UConn NRCA Projects (2021). University of Connecticut's Natural Resources Conservation Academy Project Filter Webpage. Available at: https://nrca. uconn.edu/projects/index.htm (Accessed April 14, 2021).

Varelas, M., Martin, D. B., and Kane, J. M. (2012). Content Learning and Identity Construction: A Framework to Strengthen African American Students' Mathematics and Science Learning in Urban Elementary Schools. Hum. Dev. 55, 319-339. doi:10.1159/000345324

Vygotsky, L. S. (1987). "Thinking and Speech," in The Collected Works of L.S. Vygotsky. Editors R. Rieber and A. Carton (New York, NY: Plenum), 39-285. Wenger, E. (1998). Communities of Practice: Learning, Meaning, and Identity. New York, NY: Cambridge University Press.

Conflict of Interest: The authors declare that the research was conducted in the absence of any commercial or financial relationships that could be construed as a potential conflict of interest.

Publisher's Note: All claims expressed in this article are solely those of the authors and do not necessarily represent those of their affiliated organizations, or those of the publisher, the editors and the reviewers. Any product that may be evaluated in this article, or claim that may be made by its manufacturer, is not guaranteed or endorsed by the publisher.

Copyright (๑) 2021 Cisneros, Simmons, Campbell, Freidenfelds, Arnold, Chadwick, Dickson, Moss, Rodriguez and Volin. This is an open-access article distributed under the terms of the Creative Commons Attribution License (CC BY). The use, distribution or reproduction in other forums is permitted, provided the original author(s) and the copyright owner(s) are credited and that the original publication in this journal is cited, in accordance with accepted academic practice. No use, distribution or reproduction is permitted which does not comply with these terms. 